\title{
Exfoliation of 2D materials by high shear mixing
}

Sonia Biccai, ${ }^{1,2}$ Sebastian Barwich, ${ }^{1,2}$ Daniel Boland, ${ }^{1,2}$ Andrey Harvey, ${ }^{1,2}$ Damien Hanlon, ${ }^{1,2}$ Niall McEvoy, ${ }^{1}$ Jonathan N Coleman ${ }^{1,2 *}$

${ }^{1}$ CRANN and AMBER research centers, Trinity College Dublin, Dublin 2, Ireland

${ }^{2}$ School of Physics, Trinity College Dublin, Dublin 2, Ireland.

*colemaj@tcd.ie (Jonathan N. Coleman); Tel: +353 (0) 18963859.

Abstract: While it has been demonstrated that large scale liquid exfoliation of graphene is possible using high-shear exfoliation, it has not yet been shown to be applicable to a broader range of layered materials. In addition, it would be useful to determine whether the mechanisms reported for shear exfoliation of graphene also apply to other 2D materials. In this work we show that previous models describing high-shear exfoliation of graphene apply to $\mathrm{MoS}_{2}$ and $\mathrm{WS}_{2}$. However, we find the minimum shear rate required to exfoliate $\mathrm{MoS}_{2}$ and $\mathrm{WS}_{2}$ to be $\sim 3 \times 10^{4} \mathrm{~s}^{-1}$, somewhat higher than the value for graphene. We also demonstrate the scalability of shear exfoliation of $\mathrm{WS}_{2}$. By measuring and then optimising the scaling parameters, shear exfoliation of $\mathrm{WS}_{2}$ is shown to be capable of reaching concentrations of $1.82 \mathrm{~g} / \mathrm{L}$ in 6 hours and demonstrating a maximum production rate of $0.95 \mathrm{~g} / \mathrm{hour}$.

\section{Introduction}

Due to its incredible properties and numerous applications there has been an explosion of interest in graphene since its discovery in 2004.[1-3] While exfoliation and monolayer production efforts initially focused on graphene, $2 \mathrm{D}$ carbon is far from the sole material of interest with focus shifting to the exfoliation of other 2D materials.[4-7] Transition metal dichalcogenides (TMD's) have seen applications in batteries, $[8,9]$ sensors $[10,11]$ and transistors,[12] an application where graphene is currently unsuitable due to its lack of a bandgap. For TMD's to find use in applications on an industrial scale, it is essential to find an operable method which allows production of large quantities of exfoliated and defect-free material. While a method has been demonstrated for graphene[13] this method has not yet been fully explored for TMD's.

Liquid phase exfoliation of 2D materials through the use of ultra-sonication has been known for some time [14] and, while this method can be applied to a variety of materials including 
TMD's,[15], sonication is not suitable for scaling to industrial production levels. Shear exfoliation of graphene was recently demonstrated as a facile and scalable production method to suit industrial scale production of graphene stabilised in the liquid phase [13]. Following the original demonstration of shear exfoliation in rotating blade mixers, this method had been extended by a number of researchers.[16-18] However, in many cases, shear exfoliation methods have been guided, at least in part by simple models.[13] It is unknown however whether the models proposed in this work specific to graphene or general to all 2D materials. Furthermore, this method has not been fully explored for alternative 2D materials.

One phenomenon which was observed during the scale up study using high shear mixing for graphene, was the apparent minimum shear rate required for exfoliation to take place. This "shear minimum" was understood using a simple model which described the shear minimum as a function of the energy cost of exfoliation:

$\dot{\gamma}_{\text {min }}=\frac{\left[\sqrt{E_{S, G}}-\sqrt{E_{S, L}}\right]^{2}}{\eta L}$

where $\dot{\gamma}_{\min }$ is this shear min, $\eta$ is the liquid viscosity, $L$ the minimum exfoliable platelet length and $E_{S, M}, E_{S, L}$ are the material and liquid surface energy respectively. N.B. for the exfoliation of $2 \mathrm{D}$ materials, the surface energy is usually taken as the surface tension (i.e. Gibbs free surface energy) minus the product of absolute temperature and surface entropy.[14] If we rearrange equation 1 for $\mathrm{L}$ it can be used to describe a minimum size for exfoliable platelets. Taking into account the fact that platelets over a certain size will be largely removed during centrifugation of the dispersions, we can obtain an expression for the mean dispersed platelet length as an average of these upper and lower limits:

$$
\langle L\rangle \approx \frac{\left[\sqrt{E_{S, G}}-\sqrt{E_{S, L}}\right]^{2}}{2 \eta \dot{\gamma}}+\frac{L_{C F}}{2}
$$

where $\mathrm{L}_{\mathrm{CF}}$ is the upper cut off of flake size due to centrifugation. Equation 2 provides potential routes to material optimisation through surface area matching, tailoring viscosity for desired platelet size and minimising the required shear rate for exfoliation to name a few and so being able to apply this theory to alternative materials would be highly desirable.

In this work we demonstrate that the theory describing shear exfoliation of 2D materials in the liquid phase can be applied to other materials. We then focus our attention on one material of interest $\left(\mathrm{WS}_{2}\right)$ and, through varying processing parameters, demonstrate that scalable 
production of $\mathrm{WS}_{2}$ can in fact be performed using high shear mixing reaching a production rate of $0.95 \mathrm{~g} / \mathrm{h}$.
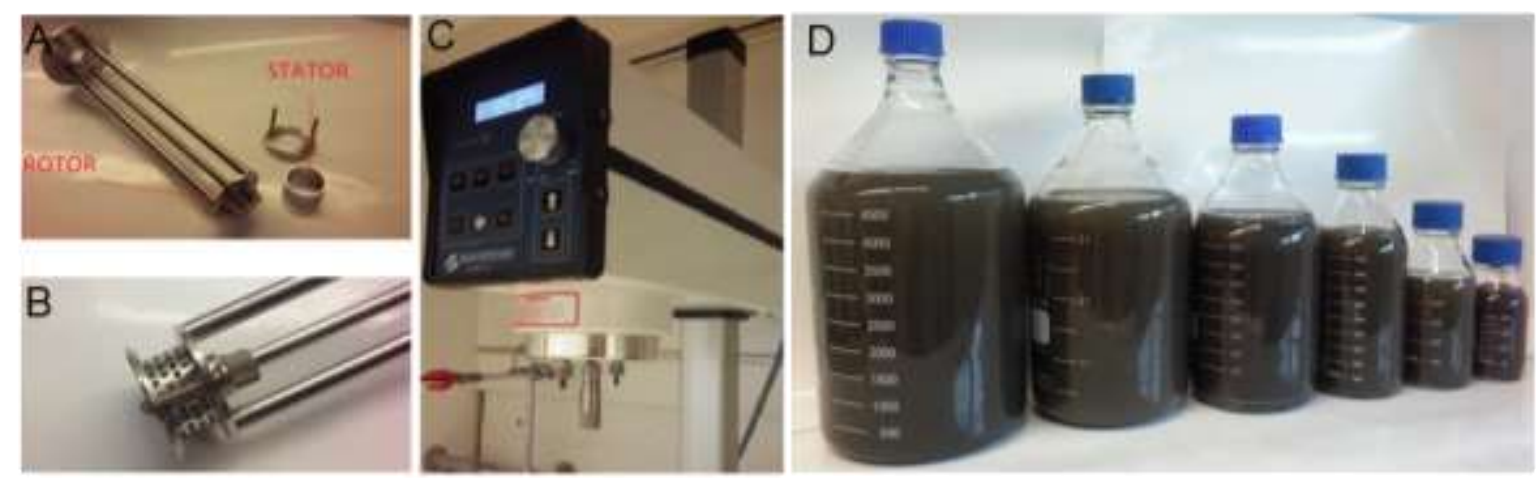

Figure 1 A) Working Head and B) Rotor-Stator Assembly. C) Silverson High Shear Mixer Used for this Work D) Large Volumes of WS 2 dispersion in Water Surfactant.

\section{Results and Discussion}

In order to demonstrate the generality of shear mixing as described previously by Paton et al [13] it is important to investigate if the theory they describe is general to all $2 \mathrm{D}$ materials or specific to graphene. We used the same high shear mixer (Silverson model L5M-A, 746 W) as in our previous work (Figure 1). All starting materials were cleaned in a pretreatment step (methods). The pre-treated materials were immersed in NMP, sheared at various shear rates, centrifuged and the resulting concentration of the supernatant was measured using UV-Vis spectroscopy (see SI). As shown in Figure 2.A, the concentration is low for small shear rates before increasing sharply above a critical value. Thus, a distinctive minimum shear rate, $\dot{\gamma}_{\text {min }}$, where exfoliation begins can clearly be seen for all materials. As observed previously, $\dot{\gamma}_{\text {min }}$ is close to $10^{-4} \mathrm{~s}^{-1}$ for graphene and is somewhat higher for $\mathrm{MoS}_{2}$ and $\mathrm{WS}_{2}$ at $\sim 3 \times 10^{4} \mathrm{~s}^{-1}$ and $\sim 3.5 \times 10^{4} \mathrm{~s}^{-1}$ respectively. This higher minimum shear rate in the TMD samples is probably partly due to the fact that nanosheets of $\mathrm{WS}_{2}$ and $\mathrm{MoS}_{2}$ tend to be smaller than graphene flakes (see below). However, another possibility is that it may also be due to slightly higher surface energies in TMD's.[15] 

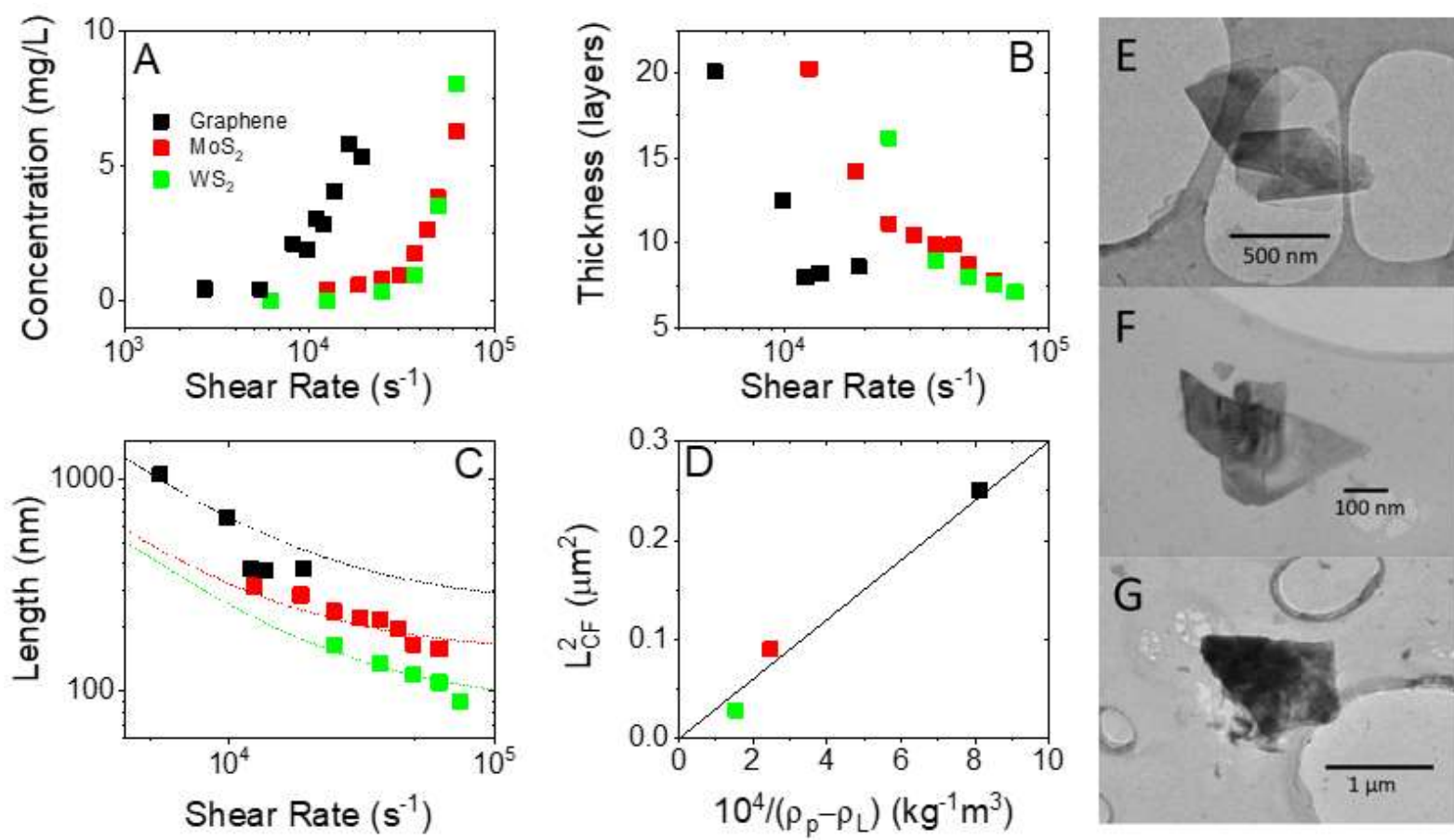

Figure 2 A) Concentration vs shear rate B) Flake thickness and C) Flake length as calculated using UV-Vis and Raman peak metrics developed by Backes et al. D) $L_{c f}$ or "cut size" as

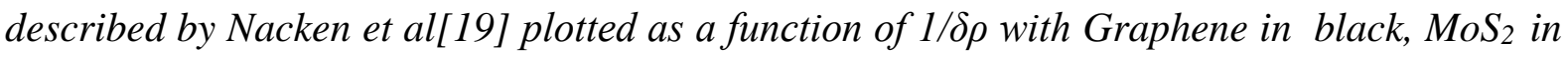
red and $W_{2}$ in green E,F,G)TEM micrographs showing exfoliated flakes of Graphene, MoS and $\mathrm{WS}_{2}$ respectively.

It has been shown previously that utilising spectroscopic metrics for nanosheet size and thickness can be an effective way to characterise LPE dispersions.[20-23] In this work we utilise the UV-Vis metrics detailed by Backes et al to characterise dispersion concentration and $\mathrm{WS}_{2}$ and $\mathrm{MoS}_{2}$ flake size and thickness, while using the Raman metrics detailed to determine graphene flake size and thickness where, for all materials, $\mathrm{L}$ is in $\mathrm{nm}$ and $\mathrm{N}$ in layers.

$$
\begin{aligned}
& N_{\text {MoS } 2}=2.3 \times 10^{36} e^{\left(-54888 / \lambda_{A}\right)} \quad N_{W S 2}=6.35 \times 10^{-32} e^{\lambda_{A} / 8.51} \quad N_{G r a}=1.04\left(I_{2 D} / I_{G^{\prime}}\right) \\
& L_{M o S 2}=\frac{3500\left(E x t_{B} / E x t_{345}\right)-0.14}{11.5-\left(E x t_{B} / E x t_{345}\right)} L_{W S 2}=\frac{2.3-\left(E x t_{235} / E x t_{290}\right)}{0.02\left(E x t_{235} / E x t_{290}\right)-0.0185} L_{G r a}=\frac{94}{\left(I_{D} / I_{G}\right)_{G^{\prime} \text { ene }}-\left(I_{D} / I_{G}\right)_{G^{\prime} \text { te }}}
\end{aligned}
$$

Using these, we can get a measure of how the platelet lateral size and thickness vary with shear rate and gain a further understanding of the exfoliation mechanics. Most notable is the dramatic decrease in thickness occurring below the shear minimum as shown in Figure 2B. Comparing the data in figure $2 \mathrm{~B}$ with that in figure $2 \mathrm{~A}$ shows the flake thickness to fall below $\sim$ ten layers at the shear minimum. 
Moving our attention to platelet length (figure 2C), it is clear that the graphene nanosheets are considerably larger than either $\mathrm{MoS}_{2}$ or $\mathrm{WS}_{2}$ nanosheets implying the difference in shear minimum between the materials described above to be size related. This graph also shows a decrease in size as a function of shear rate. The data has been fitted to equation 2 (for NMP $\eta$ $=1.7 \mathrm{mPas}$ ) with each data set being consistent with similar nanosheet surface energies of $\mathrm{E}_{\mathrm{S}, \mathrm{L}}$ $\sim 73 \mathrm{~mJ} / \mathrm{m}^{2}$ and a solvent surface energy of $\mathrm{E}_{\mathrm{S}, \mathrm{M}}=71.5 \mathrm{~mJ} / \mathrm{m}^{2}$. However, we found a significant difference between values of $\mathrm{L}_{\mathrm{CF}}$ for the different materials: 500, 300 and $170 \mathrm{~nm}$ for graphene, $\mathrm{MoS}_{2}$ and $\mathrm{WS}_{2}$ respectively.

We note that $\mathrm{L}_{\mathrm{CF}}$ is a measure of the largest flake size retained after centrifugation. An approximate expression for this parameter has been presented by Nacken et al:[19]

$$
L_{C F}=\sqrt{\frac{18 \eta}{\left(\rho_{P}-\rho_{L}\right) \omega^{2} t} \ln \left(r_{2} / r_{1}\right)}
$$

where $\eta$ is solvent viscosity, $\rho_{\mathrm{P}, \mathrm{L}}$ are platelet and liquid density respectively, $\omega$ is rotational speed, $t$ centrifugation time and $r_{1,2}$ are the minimum and maximum radii of the samples placed in the centrifuge rotor. This equation implies that the variation of $\mathrm{L}_{\mathrm{CF}}$ is simply due to variations in material density. Plotting $\left(\mathrm{L}_{\mathrm{CF}}\right)^{2}$ vs $1 /\left(\rho_{P}-\rho_{L}\right)$ in Figure $2 \mathrm{D}$ we find a very good agreement with a straight line fit, albeit with slope different by a factor of 5 from the theoretically predicted slope.

Having seen that the theory describing shear exfoliation is maintained for alternative materials, we focus our attention on scaling up $\mathrm{WS}_{2}$, a material of considerable interest, and in doing so transfer from solvent based exfoliation to exfoliation in water/surfactant.

Before attempting to optimise parameters, it is important to reconfirm that shear exfoliation in water surfactant produces nanosheets of similar quality. With this in mind, a preliminary sample was produced in order to verify the quality of the nanoparticles, shearing $20 \mathrm{~g} / \mathrm{L}$ of $\mathrm{WS}_{2}$ in $300 \mathrm{ml}$ water with sodium cholate $0.8 \mathrm{~g} / \mathrm{L}$ for 90 minutes at $9000 \mathrm{rpm}$ (referred to as a "standard" sample). TEM analysis was performed on the standard sample finding the nanosheet mean length to be $\sim 56 \mathrm{~nm}$ (Fig. 3. A, B, C). Using Raman analysis, we confirmed that the flakes were, in fact $\mathrm{WS}_{2}$ (Fig.3.D). 

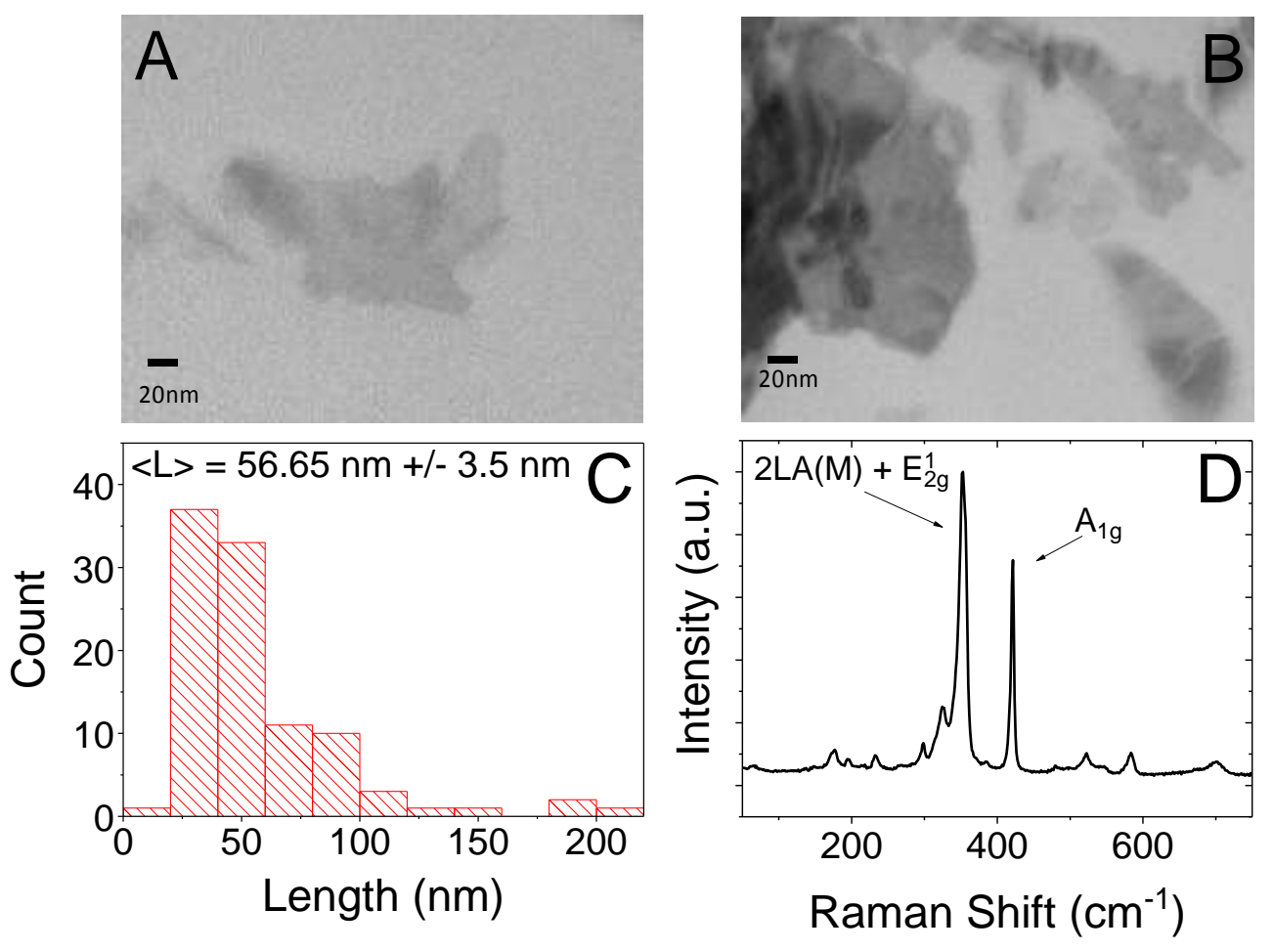

Figure 3 A-B) TEM image of nanosheets prepared from a standard sample, showing well exfoliated flakes of $W_{2}(C)$ Histogram of nanosheet length from TEM image analysis D) Raman spectrum of standard sample of $W S_{2}$ in water and sodium cholate showing the typical $W S_{2}$ peaks: in plane $E_{12 g}$ mode $(\sim 350 \mathrm{~nm})$ and out of plane $A_{1 \mathrm{~g}} \operatorname{mode}(\sim 420 \mathrm{~nm})$.

It is highly important to know which parameters are most crucial for $\mathrm{WS}_{2}$ nanosheet production with the aim to have the maximum concentration and production rate. Properties of shearmixed dispersions have a tendency to scale with processing parameters as power laws [24]. In our case, because the diameter (D) of the shear head has been kept constant, the concentration can only scale with the initial concentration of layered material $\left(\mathrm{C}_{\mathrm{i}}\right)$, the rotation rate $(\mathrm{N})$, the liquid volume $(\mathrm{V})$ and the mixing time $(\mathrm{t})$

$C \propto C_{i}^{\chi} N^{n} V^{v} t^{\tau}$

To understand the scale-up process, it is necessary to confirm this type of behaviour and to identify the exponents. For example, because the production rate is given by

$$
P_{R}=\frac{C V}{t} \propto C_{i}^{\chi} N^{n} V^{v+1} t^{\tau-1}
$$

It is clear that not only do we need $\chi, \mathrm{n}$ and $\tau$ to be large but it is imperative that $\mathrm{v}>-1$ if increasing volume is to be a relevant way to increase $P_{R}$. 
During the study, each parametric dependence was investigated in order to find each exponent and produce an equivalent final equation. In each study, concentration and mean thickness (in layers) was found using UV-vis spectroscopy (Fig.4.A).

In optical analysis the baseline is very important. In this work, the sample preparation means the exact concentration of $\mathrm{NaC}$ in the final dispersion is unknown, so we instead prepare the baseline with DI water alone. Due to the fact $\mathrm{NaC}$ absorbs at lower wavelengths, it would not be possible to use the formulas obtained by Backes et al. [22] for concentration and thickness values. For this reason, an extinction coefficient at a different wavelength needed to be used. We considered the graph of the extinction coefficient as a function of the extinction ratio $\left(\mathrm{Ext}_{\mathrm{A}} / \mathrm{Ext}_{290}\right)$ obtained by Backes et al. [22] and modified it to use a higher wavelength (350 $\mathrm{nm}$ ) and obtained the extinction coefficient from this new fitted graph (SI : S3). From this graph we can obtain the extinction coefficient, where $\varepsilon=-9.73+54.67\left(\operatorname{Ext}_{\mathrm{A}} / \mathrm{Ext}_{350}\right)^{0.757} \mathrm{~L} \mathrm{~g}^{-}$ ${ }^{1} \mathrm{~cm}^{-1}$. Similarly, the metric used to calculate platelet size had to be modified to utilize the sample extinction at $365 \mathrm{~nm}$ and $465 \mathrm{~nm}$ (SI : S4) yielding;

$\mathrm{L}=\left(3.69-\left(\operatorname{Ext}_{365} / \mathrm{Ext}_{465}\right)\right) /\left(0.011 \times\left(\operatorname{Ext}_{365} / \mathrm{Ext}_{465}\right)+0.0011\right)$ where $\mathrm{L}$ is in $\mathrm{nm}$. The metric for $\mathrm{WS}_{2}$ platelet thickness however did not need to be modified and was used again here.

The first parameter that was varied is the surfactant concentration. In this case, samples were prepared changing the concentration of $\mathrm{NaC}$ in the pre-treatment step and keeping all the other parameters constant. UV-vis analysis was performed on each sample and it was possible to calculate the concentration and the thickness of $\mathrm{WS}_{2}$ particles. The graph below shows that the dependency of nanosheet concentration displays a peak, reaching a maximum at $1.2 \mathrm{~g} / \mathrm{L}$ (Fig.4.C). This does not follow a power law dependency expected for the other parameters, however, from the data we can select the $\mathrm{NaC}$ concentration that yields the highest concentration in order to maximise $\mathrm{WS}_{2}$ production. By analysing (Fig.4.D), we can determine the dependence of nanoparticle size on the $\mathrm{NaC}$ concentration. Indeed, we notice the particle thickness decreases as the $\mathrm{NaC}$ concentration increases, this is in line with what has been observed previously for $\mathrm{MoS}_{2}$ exfoliation in surfactant.[25] Nanosheet thickness, $\langle\mathrm{N}\rangle$, within a standard sample was measured also by AFM and the histogram is shown in fig.5.A. 

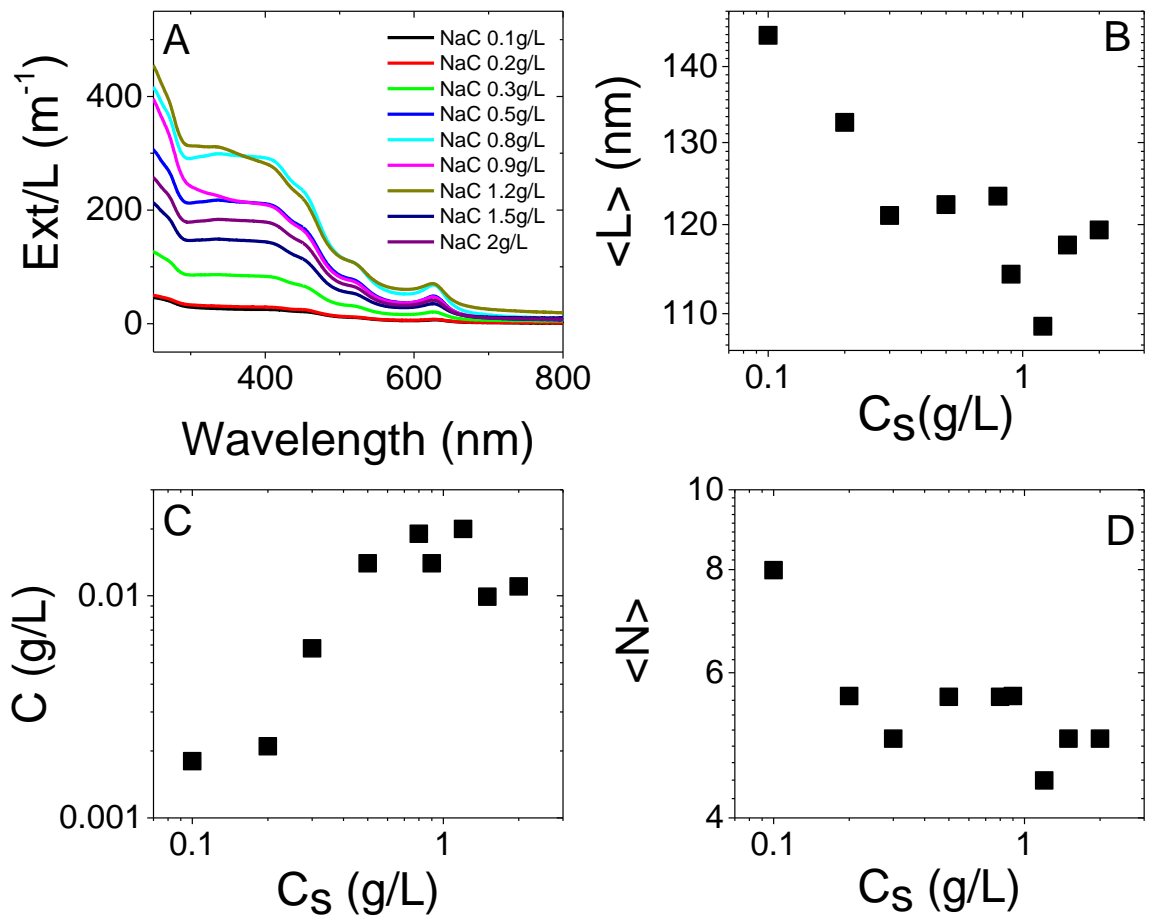

Figure 4 A) Uv-vis spectra of $W S_{2}$ for various surfactant concentrations. B) WS 2 length, (C) $W S_{2}$ concentration and $(D) W S_{2}$ thickness as a function of $N a C$ concentration.

In order to find the exponents for the scale up equation, rotation rate, $\mathrm{N}$, initial $\mathrm{WS}_{2}$ concentration, $\mathrm{C}_{\mathrm{i}}$, sample volume, $\mathrm{V}$, and shear time, $\mathrm{t}$, were varied independently keeping the other parameters constant. To find the exponent relative to the rotation rate, $\mathrm{N}$, the sample preparation followed the same procedure in both pre-treatment and shearing stage. The graph confirms as we increase the shear rate, the concentration of the resulting dispersion increases. We can also see that the concentration is steady from $6 \times 10^{4}$ to $10.6 \times 10^{4} \mathrm{~s}^{-1}$ and then rises considerably. Calculating the slope of the increasing range we find the value of the exponent $\mathrm{n}$ $=7.15$ (Fig.5.B), which is significantly larger than that seen for graphene $\left(\mathrm{n}_{\mathrm{Gra}}=1.13\right)$. Similarly, as the initial $\mathrm{WS}_{2}$ concentration is increased the final concentration increases. This increase is of the form, $C \propto C_{i}^{\chi}$, and fitting this to the data was found to be $\chi=1$, (Fig.5.C) which corresponds to the same value found for graphene. To allow as wide a range of volumes as possible to be studied, four different types of beakers were used for the volume study (600 $\mathrm{mL}, 1 \mathrm{~L}, 2 \mathrm{~L}$ and $5 \mathrm{~L}$ ) In this case, the volume study shows how concentration decreases when volume increases (Fig.5.D). The slope's value (-0.84) does not correspond exactly to the value found for graphene in NMP (N-Methyl-2-Pyrrolidone) [13] but, importantly is >-1 and so will facilitate scale up (see above). The last parameter study is the mixing time, t. This study 
revealed that the $\mathrm{WS}_{2}$ concentration increases with the time according to a power law and the exponent was found to be $\tau=0.55$ (Fig.5.E), slightly lower, but reasonably comparable to the exponent seen for graphene $\left(\mathrm{t}_{\mathrm{Gra}}=0.66\right)[13]$ as would be expected if the exfoliation process does not differ significantly. These parameters mean the overall scaling of concentration is:

$$
C \propto C_{i}^{1} N^{7.15} V^{-0.84} t^{0.55}
$$

Plotting the concentration values obtained previously as a function of this equation we obtained a linear trend (Fig.5.F); as expected this shows a slope equal to 1, which demonstrates the scalability of $\mathrm{WS}_{2}$ exfoliation using shear mixing.
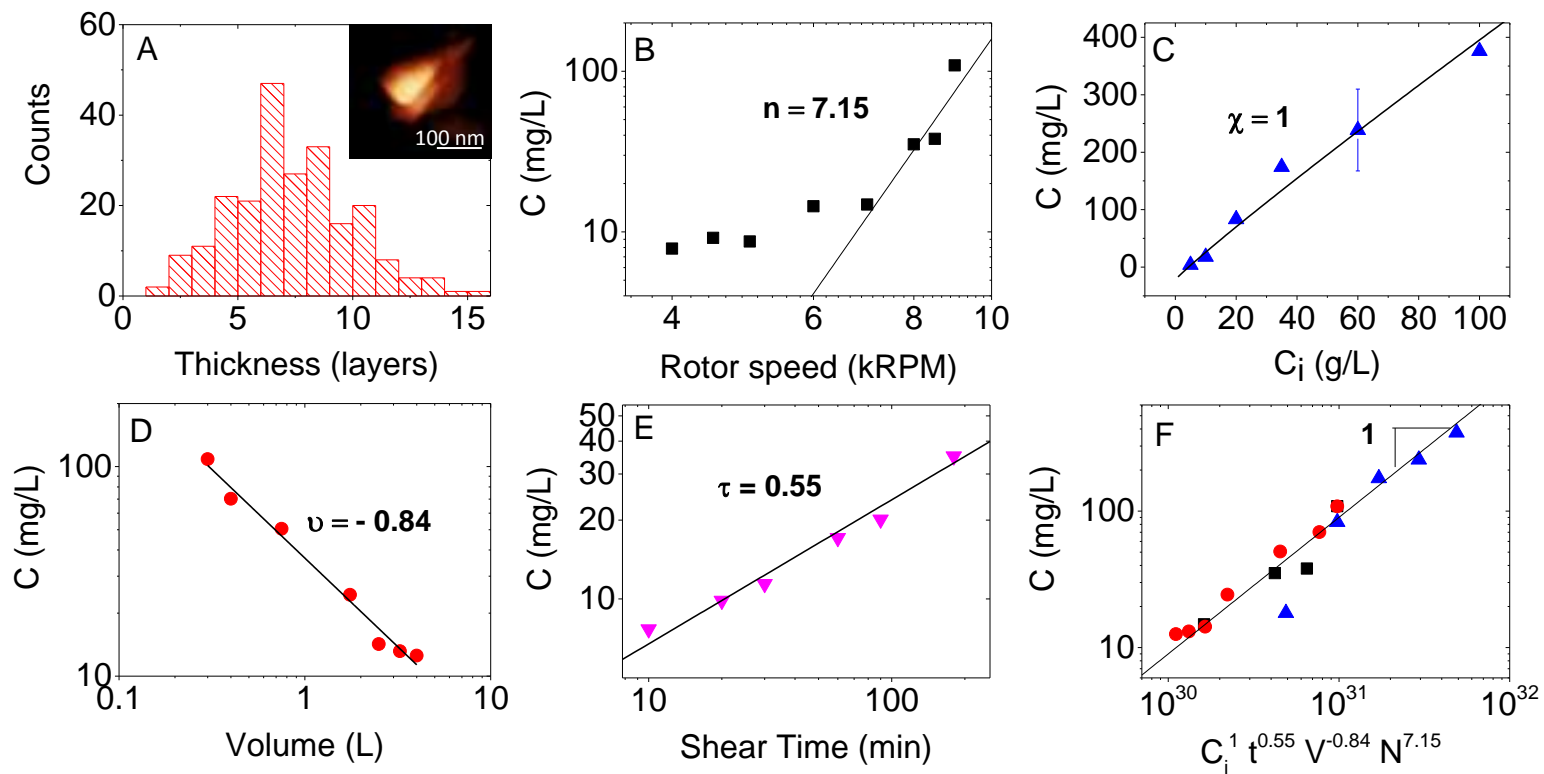

Figure 5 (A) AFM nanosheet thickness histogram and representative image of $W_{2}$ nanosheet prepared in water and $\mathrm{NaC}$ from a standard sample with mean nanosheet thickness of 6 layers (SI : Table 2). $W S_{2}$ concentration as a function of $(B)$ shear rate, $(C)$ Initial concentration of $W S_{2},(D)$ volume and $(E)$ shear time. $(F)$ Concentration of $W S_{2}$ as a function of the scaling equation determined by variation of processing parameters, data taken from Figs B-D with symbols and colours kept consistent. N.B. The data from $E$ was not included in $F$ as the exfoliation procedure was slightly different to the other samples.

Using this data, we selected parameters in order to maximise the final $\mathrm{WS}_{2}$ concentration. These parameters were; $\mathrm{V}=300 \mathrm{~mL} ; \mathrm{C}_{\mathrm{i}}=100 \mathrm{~g} / \mathrm{L} ; \mathrm{t}=6$ hours; $\mathrm{N}=9000 \mathrm{rpm}$ (Shear Rate $=13.7 \times 10^{4}$ $\mathrm{s}^{-1}$ ). The UV-vis spectra were analysed, showing a concentration of $1.82 \mathrm{~g} / \mathrm{L}$ with the mean particle thickness of approximately 6 layers and with $\langle\mathrm{L}\rangle=150 \mathrm{~nm}$. This compares favourably 
with $\mathrm{WS}_{2}$ exfoliated in water/surfactant using an ultra-sonic tip (0.77 g/L),[26] illustrating the usefulness of optimising the shear mixing parameters.

Another parameter of interest and, for commercial production, the most important parameter, is the production rate $P_{R}=C V / t$. Here, $C$ is the concentration of exfoliated material, $V$ is the volume and $\mathrm{t}$ is the shear time. Looking at the formula, we notice it is necessary to use large volumes and a short period of time to maximise $P_{R}$. To maximise the $P_{R}$ value, shear rate and initial $\mathrm{WS}_{2}$ concentration were kept the same as the high concentration sample, but volume and shear time were changed to $1.75 \mathrm{~L}$ and 10 minutes, respectively. These values are not necessarily the most optimised values and were also selected due to practicality, material availability and space concerns. Again, the sample was analysed using UV-Vis and resulted in a production rate of $0.95 \mathrm{~g} / \mathrm{h}$ with the platelets approximately 8 layers in thickness and a concentration of $0.087 \mathrm{~g} / \mathrm{L}$. While this is lower than that reported for graphene $\mathrm{P}_{\mathrm{R}, \mathrm{Gra}}=1.44$ $\mathrm{g} / \mathrm{h},[13]$ this value is merely the largest lab demonstrable value and could be further improved with increasing sample volumes/shear rates. We believe that this is the highest production rate value ever reported for $\mathrm{WS}_{2}$.

However, it is worth noting that this production rate is relatively low on an absolute scale. To be commercialised, this rate would have to be increased by more than a thousand-fold. Although some of this increase could be met by simply scaling the volume of the mixing vessel, it is likely that other improvements such as increasing rotor speed (and hence shear rate) will be necessary. A related issue is the yield of this process which is currently quite low at $<1 \%$. It will be necessary to increase this yield. There are a number of ways to achieve this with small increases achievable by slightly modifying the existing process (changing surfactant, using more efficient shear head, increasing rotor speed). However, larger increases in yield could be achieved by changing the method of generation of shear. This has been demonstrated by a number of groups who have significantly increased the shear rate by moving to wet jet milling[16] or microfluidisation.[17] As a result of the low yield, the dispersions need to be centrifuged to separate graphene from unexfoliated graphite. While the collected graphite can be recycled to increase the overall yield, the need to centrifuge adds significant inefficiencies to the process. Any commercial process would need avoid centrifugation by including a continuous, efficient separation process.

\section{Conclusions}


Here we show that shear exfoliation is generally applicable to a range of layered materials. Furthermore, it has been shown that the theory describing shear exfoliation describes the exfoliation of $\mathrm{MoS}_{2}$ and $\mathrm{WS}_{2}$ with only minor differences between these materials, namely platelet lateral size and shear min, and graphene, which has been previously studied.

These differences appear to depend largely on material density and scale closely in line with theory described by Peukert et al. That effective exfoliation of these materials depends strongly on material density, implies that optimised centrifugation techniques are vital to improving dispersion uniformity and quality with respect to mean platelet thickness.

The broad applicability of high shear exfoliation has been demonstrated further by applying the method to demonstrate the scalability of $\mathrm{WS}_{2}$ production. Shear exfoliation has been demonstrated to be affective at exfoliation of $\mathrm{WS}_{2}$ when utilising water/surfactant stabilisation in place of solvents.

Through variation of key parameters, it has been demonstrated that shear exfoliation is a suitable method to produce large quantities of exfoliated $\mathrm{WS}_{2}$. Having determined these scaling parameters, they have been utilised to obtain two maximised cases, concentration; reaching $1.82 \mathrm{~g} / \mathrm{L}$ in 6 hours, and production rate; producing $0.95 \mathrm{~g} /$ hour of material during a 15 -minute exfoliation cycle.

\section{Materials and Methods}

\section{Materials}

All chemicals were purchased from Sigma Aldrich unless otherwise stated. Dispersions were prepared using a high shear laboratory mixer (Silverson model L5M-A, $746 \mathrm{~W}$ ). It is supplied with a standard mixing assembly, comprising a frame and base plate holding a four bladed rotor and a stator with 96 square perforations $(2 \times 2 \mathrm{~mm})$ which is used for this work. The rotorstator gap for the assembly used was $\sim 110 \mu \mathrm{m}$. Centrifugation was performed using a Thermo Scientific centrifuge (Heraeus Megafuge 16) using a TX-400 swinging bucket rotor (max 5000 rpm) and a Hettich Mikro 220R centrifuge using a high speed rotor for size selection (up to 18 $\mathrm{krpm})$. Optical absorbance spectroscopy was performed with samples held in a $4 \mathrm{~mm}$ path length quartz cuvette using a UV-Vis-NIR spectrophotometer (Varian Cary 500) in $0.5 \mathrm{~nm}$ increments. Raman was performed using a Horiba Jobin Yvon LabRAM-HR using a 100× objective lens with a $633 \mathrm{~nm}$ laser excitation. TEM samples were prepared on holey carbon 
grids and was performed using a JEOL 2100 operated at $200 \mathrm{kV}$ and a FEI Titan TEM operated at $300 \mathrm{kV}$.

\section{Methods}

In this work, dispersion preparation had two steps, pre-treatment (to remove any material impurities present) and final shearing. During pre-treatment each material was sheared at 50 $\mathrm{g} / \mathrm{L}$ in IPA for 3 hours at $4500 \mathrm{rpm}$. The material was filtered through a fine pore filter membrane (nylon 0.045 micron) and dried overnight in a vacuum oven at $50{ }^{\circ} \mathrm{C}$. For final shearing, samples were prepared in various solvents at a starting concentration of $50 \mathrm{~g} / \mathrm{L}$ at various shear rates for 1 hour. These samples were all centrifuged using the same regime, 2000 rpm for 5 hours 45 minutes using the swinging bucket rotor. In order to ascertain material concentrations, extinction coefficients extracted from work performed by Backes et al were used[20-22]. Graphene samples were filtered onto a fine pore membrane (anodisc, 0.02 micron) and the resulting film was analysed using Raman. TMD samples were transferred from NMP to water surfactant $(1 \mathrm{mg} / \mathrm{ml})$ by centrifuging at $16,000 \mathrm{rpm}$ (using the high speed rotor) for 1 hour and re-dispersing the material via bath sonication before being analysed using UV-Vis. The concentration, length and thickness of graphene and the TMD's were ascertained using metrics based on Raman and UV-Vis spectra respectively.[20-22] The extinction coefficients (SI : Table 3) used were all obtained from the methods describing the length and thickness metrics for the sake of consistency.

During optimisation, dispersion/exfoliation of $\mathrm{WS}_{2}$ again comprised of two stages; a pretreatment stage (PT) to remove impurities present in the material, however with an aim to avoid toxic solvents the samples were prepared solely in water/surfactant. After that, the dispersion was left overnight to settle, before the supernatant was removed. The sediment was then redispersed in fresh DI water, and the dispersion was subjected to the final shearing. In the pretreatment step, two parameters, shear time (90 minutes) and shear rate $\left(6000 \mathrm{rpm}=9.1 \cdot 10^{4} \mathrm{~s}^{-}\right.$ ${ }^{1}$ ), were kept constant for the entire series. During the subsequent optimisation all shear parameters were varied keeping other parameters constant.

The standard sample was prepared with the following mixing parameters: a $32 \mathrm{~mm}$ rotor diameter (D), initial $\mathrm{WS}_{2}$ concentration $\left(\mathrm{C}_{\mathrm{i}}\right)$ of $20 \mathrm{mg} \mathrm{ml}^{-1}$, a $\mathrm{NaC}$ concentration $\left(\mathrm{C}_{\mathrm{s}}\right)$ of $0.8 \mathrm{mg}$ $\mathrm{ml}^{-1}$, a shearing time (t) of 90 minutes, a rpm of 9000 (shear rate $=13.7 \cdot 10^{4} \mathrm{~s}^{-1}$ ) for the shearing stage $(\mathrm{N})$, and a $200 \mathrm{~mL}$ volume $(\mathrm{V})$ for both pre-treatment and shearing. 
The volume was varied in both the PT and the shearing step, and the rotor diameter was kept constant $(32 \mathrm{~mm})$. The dispersion preparation was followed by size selection using the centrifuge speed (2-10 krpm) to select a particular range of particle dimension. The supernatant was collected after the shear mixing and centrifuged in $50 \mathrm{~mL}$ vials at $2000 \mathrm{rpm}$ for two hours; the supernatant was then re-collected and further centrifuged at 10,000 rpm for two hours. The resulting sediment was then collected, re-dispersed in water, and analysed.

\section{References}

1. Novoselov, K.S., et al., Electric Field Effect in Atomically Thin Carbon Films. Science, 2004. 306(5696): p. 666-669.

2. Keeley, G.P., et al., Electrochemical ascorbic acid sensor based on DMF-exfoliated graphene. Journal of Materials Chemistry, 2010. 20(36): p. 7864-7869.

3. Pumera, M., Electrochemistry of graphene: new horizons for sensing and energy storage. The Chemical Record, 2009. 9(4): p. 211-223.

4. Nicolosi, V., et al., Liquid Exfoliation of Layered Materials. Science, 2013. 340(6139).

5. Song, J.-G., et al., Layer-Controlled, Wafer-Scale, and Conformal Synthesis of Tungsten Disulfide Nanosheets Using Atomic Layer Deposition. ACS Nano, 2013. 7(12): p. 1133311340.

6. Ovchinnikov, D., et al., Electrical Transport Properties of Single-Layer WS2. ACS Nano, 2014. 8(8): p. 8174-8181.

7. Tao, H.C., et al., Scalable exfoliation and dispersion of two-dimensional materials - an update. Physical Chemistry Chemical Physics, 2017. 19(2): p. 921-960.

8. Feng, C., et al., Synthesis of tungsten disulfide (WS2) nanoflakes for lithium ion battery application. Electrochemistry Communications, 2007. 9(1): p. 119-122.

9. Wang, J.-Z., et al., Development of MoS2-CNT Composite Thin Film from Layered MoS2 for Lithium Batteries. Advanced Energy Materials, 2013. 3(6): p. 798-805.

10. Park, M., et al., MoS2-Based Tactile Sensor for Electronic Skin Applications. Advanced Materials, 2016. 28(13): p. 2556-2562.

11. Wang, Q.H., et al., Electronics and optoelectronics of two-dimensional transition metal dichalcogenides. Nature Nanotechnology, 2012. 7: p. 699.

12. Kelly, A.G., et al., All-printed thin-film transistors from networks of liquid-exfoliated nanosheets. Science, 2017. 356(6333): p. 69-73.

13. Paton, K.R., et al., Scalable production of large quantities of defect-free few-layer graphene by shear exfoliation in liquids. Nat. Mater., 2014. 13(6): p. 624-630.

14. Hernandez, Y., et al., High-yield production of graphene by liquid-phase exfoliation of graphite. Nat Nano, 2008. 3(9): p. 563-568.

15. Cunningham, G., et al., Solvent Exfoliation of Transition Metal Dichalcogenides: Dispersibility of Exfoliated Nanosheets Varies Only Weakly between Compounds. ACS Nano, 2012. 6(4): p. 3468-3480.

16. Del Rio Castillo, A.E., et al., High-yield production of $2 D$ crystals by wet-jet milling. Materials Horizons, 2018. 5(5): p. 890-904.

17. Karagiannidis, P.G., et al., Microfluidization of Graphite and Formulation of Graphene-Based Conductive Inks. Acs Nano, 2017. 11(3): p. 2742-2755.

18. Shinde, D.B., et al., Shear Assisted Electrochemical Exfoliation of Graphite to Graphene. Langmuir, 2016. 32(14): p. 3552-3559.

19. Nacken, T.J., et al., Delamination of graphite in a high pressure homogenizer. RSC Advances, 2015. 5(71): p. 57328-57338. 
20. Backes, C., et al., Spectroscopic metrics allow in situ measurement of mean size and thickness of liquid-exfoliated few-layer graphene nanosheets. Nanoscale, 2016. 8(7): p. 4311-4323.

21. Backes, C., et al., Edge and confinement effects allow in situ measurement of size and thickness of liquid-exfoliated nanosheets. Nat Commun, 2014. 5.

22. Backes, C., et al., Production of Highly Monolayer Enriched Dispersions of Liquid-Exfoliated Nanosheets by Liquid Cascade Centrifugation. ACS Nano, 2016. 10(1): p. 1589-1601.

23. Backes, C., et al., Guidelines for Exfoliation, Characterization and Processing of Layered Materials Produced by Liquid Exfoliation. Chemistry of Materials, 2017. 29(1): p. 243-255.

24. Hall, S., et al., Scaling up of Silverson rotor-stator mixers. Can. J. Chem. Eng., 2011. 89: p. 1040-1050.

25. Varrla, E., et al., Large-Scale Production of Size-Controlled MoS2 Nanosheets by Shear Exfoliation. Chemistry of Materials, 2015. 27(3): p. 1129-1139.

26. Guardia, L., et al., Production of aqueous dispersions of inorganic graphene analogues by exfoliation and stabilization with non-ionic surfactants. RSC Advances, 2014. 4(27): p. 1411514127. 